Check for updates

Cite this: J. Mater. Chem. A, 2020, 8 , 656

Received 2nd October 2019

Accepted 19th November 2019

DOI: 10.1039/c9ta10881e

rsc.li/materials-a

\section{Genetically engineered protein based nacre-like nanocomposites with superior mechanical and electrochemical performance $\uparrow$}

\author{
Prodyut Dhar, (D)*ac Josphat Phiri, (D) a Géza R. Szilvay, ${ }^{\mathrm{b}}$ Ann Westerholm-Parvinen, ${ }^{\mathrm{b}}$ \\ Thaddeus Maloney ${ }^{a}$ and Päivi Laaksonen*ad
}

\begin{abstract}
The molecular engineering of proteins at the atomistic scale with specific material binding units and the introduction of designed functional-linkers provides a unique approach to fabricate genetically modified high performance and responsive biomimetic composites. This work is inspired by a tough biological material, nacre, which possesses a hierarchical 'brick-mortar' architecture containing multifunctional soft organic molecules, which plays a significant role in improved mechanical properties of composites. A bio-inspired composite, using a resilin-based hybrid protein polymer with selective binding motifs for reduced graphene oxide (RGO) and nanofibrillated cellulose (NFC), was developed. The adhesive and elastic domains of fusion proteins show a synergistic effect with improvement in both the strength and toughness of synthetic nacre. We observed that the hybrid protein could act as a spacer molecule tuning the ion sorption and transport across the inter-layers of NFC/RGO depending on the processing conditions. Interestingly, the protein complexed freestanding solid-state films showed negligible internal resistance and improved supercapacitance suitable for flexible electronic devices. The protein-mediated binding of NFC and RGO reduces the resistance arising from poor electrode/electrolyte interfaces, which is difficult to achieve through conventional routes. The current biosynthetic route for engineering proteins provides a novel prospect to develop materials programmed with desired properties, depending on target applications.
\end{abstract}

\section{Introduction}

Biologically engineered natural materials contain highly ordered molecular building blocks, processed with precision, which is difficult to mimic in artificial systems over multiple scales. Some of the well-known toughest biological materials such as bone, pearl, and silk have in common self-assembled layers of aligned hard reinforcing and dissipating components. ${ }^{1}$ At the molecular scale, pearls found in mollusk shells contain highly aligned aragonite crystal layers chemically composed of calcium carbonate, embedded within a complex mixture of proteins (chitin derivatives). ${ }^{2}$ The hard components (aragonite layers) are glued with soft organic lubricant interconnections which helps in redistribution of strain, resulting in the formation of materials that are both extremely tough as well

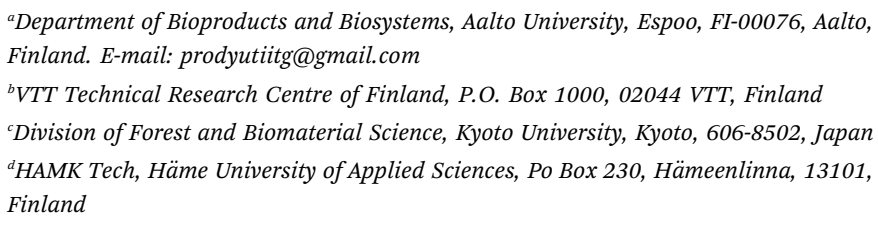
$\dagger$ Electronic supplementary information (ESI) available. See DOI: 10.1039/c9ta10881e as strong. ${ }^{3}$ However, along with the interesting morphological characteristics, living materials also provide functionality (such as actuation, motion, etc.) triggered through an externally applied biological stimulus (e.g. pH, temperature or light). Learning from nature, in the present study, strategic engineering of composites with genetically modified designer proteins is carried out to fabricate artificial nacre with highperformance mechanical as well as stimuli-responsive electrochemical behavior.

The locomotion (jumping or flying) and sound generation in insects are the coherent functions of muscle and resilin proteins, which show a unique energy storage and release mechanism. ${ }^{4}$ They are elastomeric proteins found in the insect cuticle forming a cross-linked network-like structure, with high resilience due to the presence of glycine and proline residues. ${ }^{5}$ Interestingly, resilin and resilin like proteins (RLPs) with their attractive chemo-mechanical properties show multi-stimuli responsive behaviour towards temperature, $\mathrm{pH}$, ions, and light. ${ }^{6}$ The stimuli-responsive characteristics of RLPs have found potential applications in biosensors, biomedical devices, and controlled drug delivery. Rec1-resilin, containing an $\mathrm{N}$ terminal domain (18 copies of a 15 amino acid repeat sequence of exon1) derived from Drosophila melanogaster, was first recombinantly expressed and studied by Elvin et al. ${ }^{5}$ The 
recent advances in molecular-cloning techniques and protein engineering have made it possible to design proteins at a molecular level with precise control on the amino acid sequence, binding motifs and functionality depending on target applications. Several studies on the development of smart polymeric composites using biomimetic proteins such as elastin, ${ }^{7}$ silk, ${ }^{8}$ resilin, ${ }^{9}$ green fluorescent proteins ${ }^{10}$ or abductin ${ }^{11}$ with responsive properties have been reported.

In this work, a RLP was modified with two adhesive proteins which have site-specific molecular recognition capabilities for NFC and RGO, respectively. The developed diblock fused protein complexes effectively mimic the matrix-mediated proteins found in biological systems. Firstly, the affinity of hybrid proteins towards a nanocellulose surface was enhanced by fusing two cellulose binding domains (CBMs), which are generally found in fungal cellulase enzymes. ${ }^{12}$ Secondly, a hydrophobin (HFBI), which is a fungal surfactant protein, was used to increase the molecular adhesiveness of fusion proteins towards the hydrophobic graphene surface. ${ }^{13}$ The fused protein complex binds selectively to the strong (tensile strength $\sim 130$ GPa) and highly conductive graphene surface and stiff NFC fibers (tensile modulus $\sim 110 \mathrm{GPa}$ ) interconnected through a highly flexible and responsive RLP sequence. The resulting nanocomposite formed with such engineered protein moieties mimics the hierarchical arrangements of aragonite sheets pooled in a chitinous matrix, ${ }^{14}$ as observed in biological nacre. Moreover, in the case of Pinctada fucata (pearl oyster) a similar class of protein-based di-block copolymers, Pif-97 and Pif-80, are known to introduce biomineralization and have a specific binding domain for aragonite and chitin residues. ${ }^{2,15}$

Graphene, due to its remarkable physicochemical and structural properties and improved functionality, has been widely integrated into artificial nacre using various selfassembly approaches. ${ }^{1}$ Several types of interfacial interactions at the molecular level based on non-covalent bonding such as ionic and hydrogen bonding and electronic $\pi-\pi$ interactions and covalent bonding may prevail independently or synergistically in fabricated graphene-based artificial nacre. However, there still remain several challenges for artificial nacre such as simultaneous improvement of the tensile modulus as well as toughness and processing on larger scales with the capability to mimic biological functions. The utilization of bio-inspired protein integrated artificial nacre may overcome these challenges, but has been seldom studied. A study on biomineralization within a chitosan matrix infiltrated with silk fibroin through an ice-templating and hot-pressing method for artificial nacre fabrication was recently reported by Mao et al. ${ }^{16}$ In another study by Cui et al., ${ }^{17}$ graphene-based nacre with synergistic effects (hydrogen and covalent bonding) was developed using dopamine, which mimics mussel adhesive proteins. Recent studies on infiltration of graphene layers with chitosan ${ }^{18}$ and pyrimidinone based supramolecular assembly ${ }^{19}$ have shown the presence of a similar type of protein-based interaction forming tough and strong composites. In our earlier reported study, we have shown that strategic designing of fusion proteins with specific recognition sites can be used to fabricate composites with improved structural properties. ${ }^{20,21}$ However, the properties of developed nanocomposites cannot be externally tuned or controlled because of the absence of any functional and responsive units in diblock proteins. In the present study, the introduction of a functional linker in the modular protein is expected to tune the microstructure of $\mathrm{NFC} / \mathrm{RGO}$ significantly, depending on processing conditions (e.g. $\mathrm{pH}$, temperature or ionic strength). To the best of our knowledge, the fabrication of stimuli-responsive protein-based artificial nacre has never been reported and is expected to show highperformance applications in structural and electronic sectors.

Inspired from the modular nature of structural or matrix proteins in biological systems, we designed a hybrid protein in which molecular recognition sites (CBM and HFBI) are connected with a functional linker (resilin-like polypeptide, RLP) through genetic engineering methods. The fusion protein forms a soft matrix adhering to NFC and RGO surfaces through molecular or colloidal-level interactions and establishing a biomimetic nacre-like morphology. Resilin generally present in insects has an energy storage function with the capability to rapidly store and release energy without fatigue..$^{22}$ The high resilience of the linker molecule coupled with stiff building blocks has shown to distinctively improve both the strength and toughness of the material. The binding interactions and the degree of intercalations of hybrid proteins, however, depend on their concentrations, NFC/RGO ratio, and processing conditions which have been studied in detail. The utilization of fusion proteins has shown to modulate the micro-architecture of NFC/RGO with pH tunable inter-layer spacing, resulting in significant variation of electrochemical charge storage capabilities. In summary, the present study proposes a biosynthetic route for modulating materials through molecular engineering of functionally active proteins and evaluate their practical applications as flexible solid-state supercapacitors.

\section{Results and discussion}

\section{Evaluation of molecular interactions between the fusion protein and NFC/RGO nanosheets}

The fusion protein was genetically engineered to include both adhesive and elastically active molecular building blocks which are connected by polypeptide linkers. The two adhesive protein binding moieties used were cellulose-binding modules (CBMs) and a class II hydrophobin (HFBI), which were interconnected to each other with a functional resilin-like polypeptide (RLP) (Fig. 1(a) and S1(a) $\dagger$ ). The developed modular protein was anticipated to self-assemble in the interlayers of RGO and NFC to create hybrid nanocomposites with functional and responsive characteristics (as shown in Fig. 1(b)). Fungal CBMs originating from cellobiohydrolase enzymes of fungus Trichoderma reesei bind specifically to cellulosic surfaces through aromatic residues and hydrogen bonding. ${ }^{23}$ CBMs are generally rich in disulfide cross linkages with the presence of cysteine bridges and are relatively smaller in size (made up of $\sim$ about 36-38 amino acid residues). ${ }^{24}$ To enhance the binding efficiency with cellulose fibers, two CBMs were connected in tandem with a non-functional small amino acid linker and are referred to as 'dCBMs' (double CBMs) in the fusion protein. The second 
(a) i. Recognition Proteins:

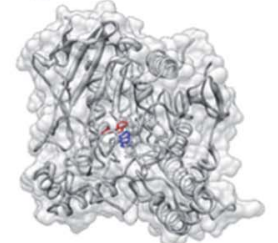

Hydrophobin

(HFBI)

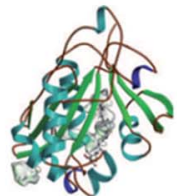

Cellulose binding modules (CBM)

\section{ii. Functional Linker: Resilin}

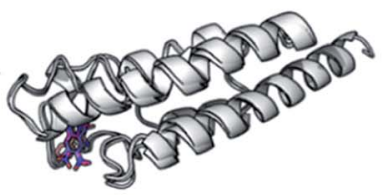

(b)

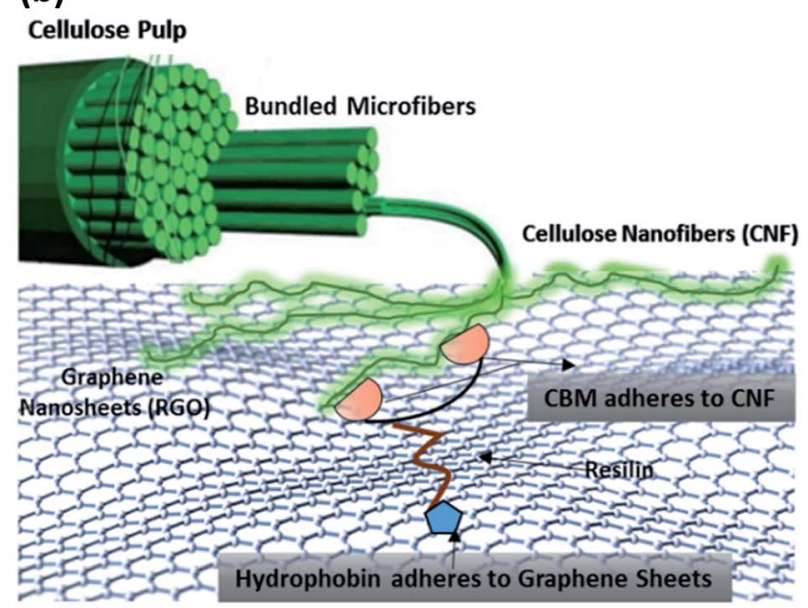

iii. Hybrid Functional Protein
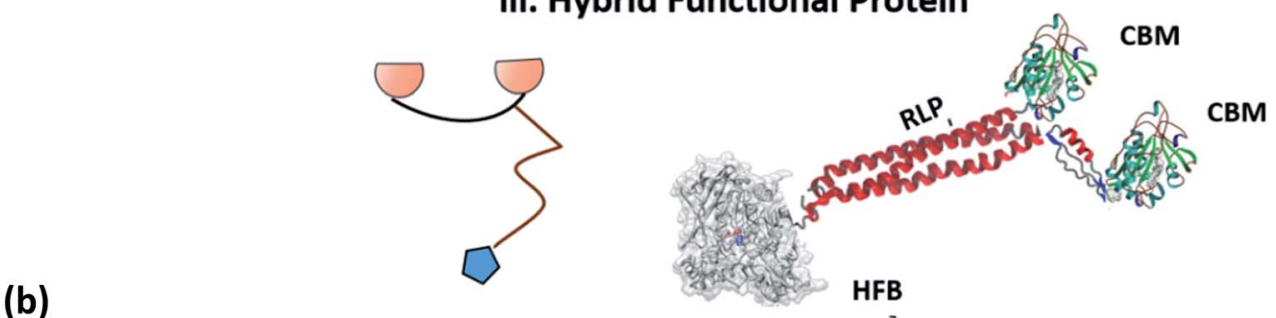

\section{(RLP) \\ Resilin-like polypeptide}

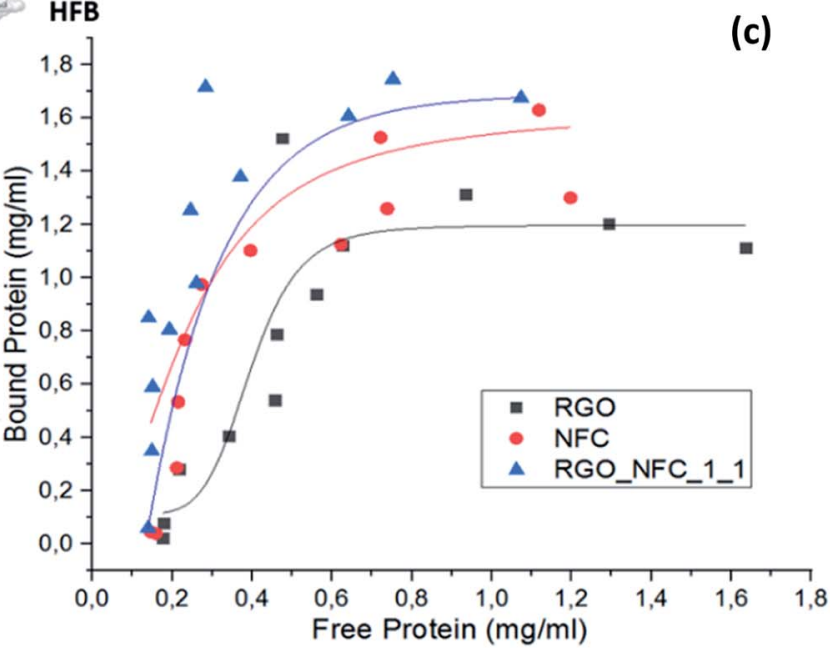

\section{Extension of the inter-layer spacing of NFC and RGO in presence of the different $\mathrm{pH}$ conditions} induced through the conformational changes of the $\mathrm{pH}$ responsive

(d)

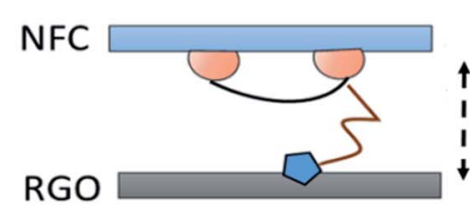

$\mathrm{pH}^{\sim} 4$

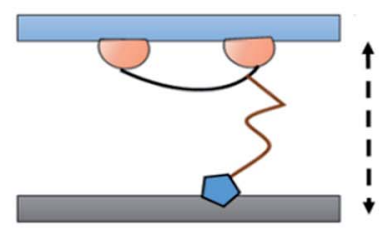

$\mathrm{pH}^{\sim} 7$

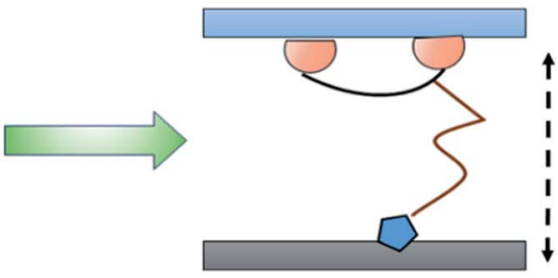

$\mathrm{pH}^{\sim} 11$

Fig. 1 A schematic representation of the self-assembled composites using the genetically engineered protein: (a) diblock protein with two recognition sites (i) connected through a functional linker, resilin-like polypeptide (RLP) (ii). The hybrid fusion protein has two functional cellulose binding modules (CBMs) which specifically bind to nanocellulose (NFC) and an amphiphilic hydrophobin (HFBI) protein which adheres to the reduced graphene oxide sheets (RGO) (iii). (b) The self-assembled fusion protein at the interface between NFC and RGO, with the two CBM sites (dCBM), adhere to cellulose through site-specific recognition and HFBI to the graphene sheets through hydrophobic interactions. (c) Binding isotherms (fitted using the Hill equation) of the fusion protein dCBM-RLP-HFB with NFC, RGO and NFC : RGO (1:1) measured at a constant concentration of $2 \mathrm{~g} \mathrm{~L}^{-1}$ and at $\mathrm{pH}$ 7. (d) At the molecular level, the functional linker RLP undergoes a transition in size under different $\mathrm{pH}$ conditions resulting in tunable inter-layer spacing between the graphene and NFC sheets.

adhesive component, hydrophobin HFBI, is slightly larger with a diameter of $\sim 3 \mathrm{~nm}$ and has an amphiphilic structure..$^{25} \mathrm{It}$ is known to form multimeric units binding specifically to graphene nanosheets through hydrophobic interactions and forming a thermodynamically stable dispersion. ${ }^{21}$ The binding modules are linked together with a functionally active elastomeric protein, resilin-like polypeptide (RLP), which has a $\mathrm{pH}^{-}$ dependent reversible conformational behavior (as shown in 
Fig. 1(c)). The RLP assumes a random coil-like structure with a resilience of $\sim 95 \%$ and a deformation capability of $\sim 300 \%$, behaving like a super-elastic rubber under hydrated conditions. ${ }^{4}$ From single molecular force spectroscopy (SMFS) studies, it was observed that the RLP assumes a coil-like morphology at $\mathrm{pH} \sim 5$ and elongates at $\mathrm{pH} \sim 11$, stretching itself completely by $\sim 40 \mathrm{~nm} .{ }^{26}$ It is further quantified that at $\mathrm{pH}$ $\sim 11$, resilin forms an entangled network-like structure resulting in a high work of rupture improving by ten orders of magnitude (in comparison to that at $\mathrm{pH} \sim 5$ ), as reported in our earlier studies. $^{26,27}$ Therefore, the self-assembled layers of such functional fusion proteins in hierarchical layers of RGO and NFC are expected to tune the macroscopic properties of the RGO/NFC nanocomposites in bulk (as presented in Fig. 1(c)).

To further understand the molecular interactions of fusion proteins, binding isotherm studies were carried out in the presence of NFC and RGO as individual components as well as in the combination of RGO/NFC (at a ratio of $1: 1$ ) (Fig. 1(d)). It was expected that the binding capacity would be affected by the concentration of species, ionic strength, $\mathrm{pH}$ of the medium and number of binding sites present in the protein used for the adsorption study. ${ }^{28}$ In the present work, the protein binding studies were carried out at constant RGO, NFC and NFC/RGO (1:1) and $\mathrm{pH} \sim 7$, under varying protein concentration and isotherms were fitted with the one site Langmuir model ${ }^{29}$ and the multiple binding site based Hill equation. ${ }^{30}$ The Langmuir isotherm showed a $B_{\max }$ of $\sim 5.8,6.1$ and $13.5 \mathrm{mg} \mathrm{g}^{-1}$ for NFC, RGO and NFC/RGO respectively but with lower confidence values $\left(R^{2}\right)$ especially for NFC/RGO. This was possibly because the developed fusion protein constructs may interact with both RGO and NFC in multiple ways, and the binding was therefore evaluated using the Hill equation (with the parameters as shown in Table S1 in the ESI $\dagger$ ), which takes into consideration the multi-site adsorption behavior. The investigation of the equilibrium dissociation $\left(K_{\mathrm{d}}\right)$ and association constants $\left(K_{\mathrm{a}}\right)$ showed that both RGO and NFC in combination have higher protein binding activity. Interestingly, both $K_{\mathrm{a}}$ and $K_{\mathrm{d}}$ values for the case of RGO were found to be significantly higher which results in lower adsorption of the protein. Such behavior is due to the presence of unbound hydrophobins providing multiple sites for reversible adsorption/desorption of RGO. ${ }^{31}$ Addition of NFC lowers the $K_{\mathrm{d}}$ values suggesting that the dCBM binds strongly to the NFC fibers. Furthermore, we examined the Hill constant, $n$, which is a critical measure to determine the nature of binding and the degree of cooperativity between the multifunctional protein and ligands. ${ }^{32,33}$ It should be noted that the multiple binding behavior arises from the linker polypeptide RLP, with varying hydrophobicity index. ${ }^{34}$ It displays hydrophobic behavior under nascent conditions but when flanked with CBMs shows hydrophilic characteristics. ${ }^{34}$ The Hill coefficient values for all the three cases were found to be $n>1$, suggesting that the fusion protein dCBM-RLP-HFB showed positive cooperative binding. It represents that once dCBM-RLP-HFB binds to the nanomaterial forming a monolayer surface, it enhances the affinity and binding energy for the surrounding ligands resulting in progressive protein adsorption. As HFBI and CBMs adhere to RGO or NFC respectively, the connecting sequence RLP undergoes conformational changes which synergistically improves the cooperative binding affinity between the two systems. A similar observation was reported in our earlier study, ${ }^{27}$ in which RLP based coacervates anchored onto the surface of graphene forming a non-continuous layer with strong adhesive behavior. Therefore, the Hill equation could be used to effectively determine the binding isotherm parameters with high regression $\left(R^{2} \sim 0.99\right)$ which confirmed the adsorption of both RGO and NFC to specific binding motifs present in the fusion protein. For the case of NFC and RGO, the maximum binding capacity of dCBM-RLP-HFB in terms of mass concentration was found to be $\sim 0.9 \mathrm{~g}$ of protein per gram of $\mathrm{RGO}, \sim 1.1 \mathrm{~g}$ of protein per gram of NFC and $\sim 1.6 \mathrm{~g}$ of protein in the presence of both NFC and RGO (at a $1: 1$ ratio). From the adsorption isotherms and evaluation of parameters, it could be concluded that binding of the modular protein to NFC/RGO was governed by multi-binding behavior with positive cooperation which helps in effective transfer of resilin-functionality to the developed nanocomposites.

\section{Understanding the effect of resilin-based fusion proteins on the physicochemical properties of RGO/NFC nanocomposites in different $\mathrm{pH}$ environments}

As described in our earlier studies, ${ }^{26}$ single molecular force spectroscopy for dCBM-RLP-HFB shows reversible swelling on changing the $\mathrm{pH}$ from 5 to 11 due to conformational changes. Therefore, the assembly of fusion proteins in the interlayers of NFC/RGO was expected to alter the layered structure on macroscopic scales. The protein dissolved in buffers with different $\mathrm{pH}$ ( $\mathrm{pH} \sim 4,7$ and 11 ) was processed into a nanocomposite film with the addition of NFC/RGO (at a mass ratio of $1: 3$ ) through a solvent-casting approach (Fig. 2).

The examination of partially oxidized RGO through XRD spectroscopy (as shown in Fig. 2(d)) showed a characteristic peak at $2 \theta=26.6^{\circ}$ representing chemically reduced graphitelike patterns (002). ${ }^{35}$ Interestingly, air-dried NFC/RGO films processed at different $\mathrm{pH}$ show characteristic peak shifting towards lower angles at $\mathrm{pH} \sim 7$ and 11 respectively, with relatively sharper intensity. The calculated $d$-spacing of the films was found to be $\sim 3.29 \AA$, $3.70 \AA$, and $3.42 \AA$ corresponding to films processed at $\mathrm{pH} \sim 4,7$ and 11, respectively. At $\mathrm{pH} \sim 4$, dCBM-RLP-HFB acquires a collapsed conformation due to which the $d$-spacing remained unaltered in comparison to RGO (with a $d$-spacing of $\sim 3.25 \AA$ ). However, with increasing $\mathrm{pH}$, resilin acquired an extended morphology with the contour length growing from $29 \mathrm{~nm}$ (at pH 5) to $64 \mathrm{~nm}$ (at pH 11), in hydrated states. ${ }^{26}$ It was assumed that adhesive domains of fusion proteins bind specifically to NFC/RGO, and the extensional fragment RLP will tune the inter-spacing depending on pH. A similar observation was found in NFC/RGO films; the extensibility between interlayers was increased by $\sim 0.35 \AA$ with increasing $\mathrm{pH}$. However, as expected the expandability of the modular protein with changing $\mathrm{pH}$ was relatively lowered when bound on the surface in the dried state of solid films (compared to hydrated conditions). Although the $d$-spacing values are related to the extensional behavior of resilin, it is difficult to 

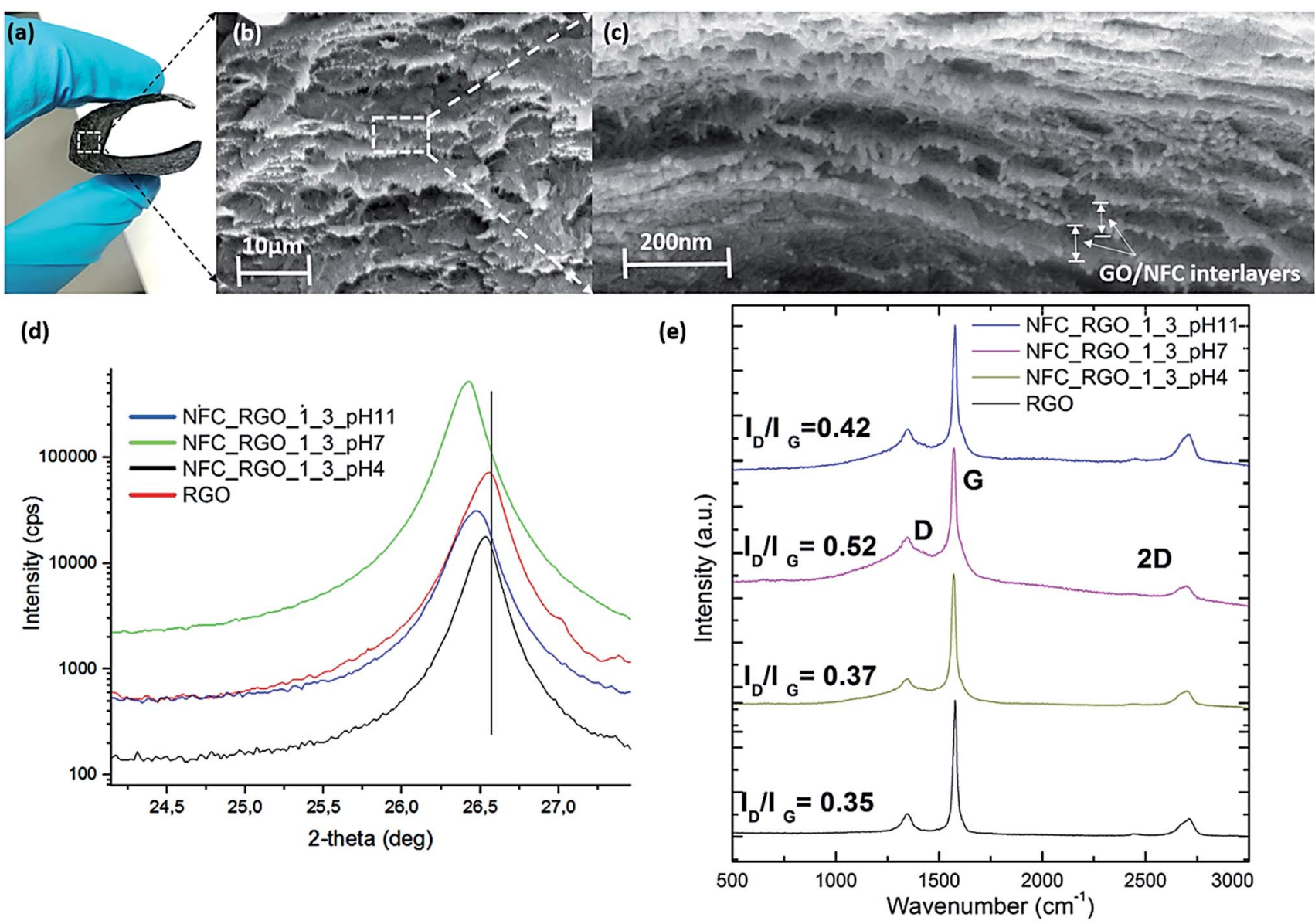

Fig. 2 Microstructure of the RGO/NFC films: (a) free-standing films with high flexibility and easy handling, (b) morphological analysis of the cross section of the films, (c) high resolution FESEM micrographs of a selected section showing the presence of a lamellar structure with the presence of the alternate layers of RGO sheets and NFC fibers (for NFC : RGO at 1: 3 processed at $\mathrm{pH} \sim 7$ with the protein added at $\sim 2.2 \mathrm{~g} \mathrm{~L}^{-1}$ ), (d) XRD patterns of the RGO/NFC films when processed in the presence of different $\mathrm{pH}$ solutions, and (e) Raman spectroscopy analysis of RGO/NFC films showing the changes in the $I_{D} / I_{G}$ ratio as a function of $\mathrm{pH}$.

quantify and correlate with the conformational changes of the RLP in the case of solid-like films. At higher $\mathrm{pH}$, the lowered $d$ spacing values are probably due to high swelling behavior of cellulose, which reduces the binding affinity and hinders the preferential interaction of cellulose with dCBM-RLP-HFB. From SMFS spectroscopic studies ${ }^{26}$ we observed that cellulose filaments under alkaline conditions were highly stretched as can be confirmed from their increased persistence length $(\sim 0.12 \pm$ $0.1 \mathrm{~nm}$ compared to other $\mathrm{pH}$ values). Along with it, both the force of adhesion and the work of rupture of fusion proteins on the cellulose surface decreased significantly, which confirms the poor binding interaction of dCBM-RLP-HFB. It is worthy mentioning that the processed NFC/RGO nanocomposite films at different $\mathrm{pH}$ preserved the tunable inter-layer spacing induced by conformational changes of the RLP even after removal of solvent. In summary, XRD studies confirm the formation of NFC/RGO films with a layered structure and expandable $d$-spacing in the range of $\sim 3.2-3.7 \AA$ depending on the $\mathrm{pH}$ of casting solution, which is expected to tune the structural as well as electrochemical properties of the developed nanocomposites.
Raman spectroscopy analysis was carried out to investigate the functionality and structural changes induced by fusion proteins in RGO nanosheets especially their degree of exfoliation when embedded in the NFC matrix. RGO shows characteristic peaks at $\sim 1349 \mathrm{~cm}^{-1}$ and $\sim 1574 \mathrm{~cm}^{-1}$ representing the $\mathrm{D}$ and $\mathrm{G}$ bands, respectively, and the peak at $\sim 2703 \mathrm{~cm}^{-1}$ corresponds to the $2 \mathrm{D}$ band $^{35}$ (shown in Fig. 2(e)). The G band signifies the in-plane vibration arising from $\mathrm{sp}^{2}$ hybridized carbon atoms present in the RGO backbone, which originates from first-order scattering of $\mathrm{E}_{2 \mathrm{~g}}$ phonons. ${ }^{36}$ The $\mathrm{D}$ band is due to out-of-plane vibrations (breathing modes of $\mathrm{sp}^{2}$ atoms) arising from structural defects in RGO and originates from TO phonons around the $K$ point of the Brillouin zone, activated by double resonance (DR). ${ }^{37}$ In Fig. $2(\mathrm{e})$, the presence of a low intensity D peak is attributed to the existence of defects on the RGO backbone introduced during the partial oxidation process (sonication in the presence of peroxides) but with a higher degree of structural order and relatively lower fractions of defects. The high intensity G-band confirms the presence of defect free graphene rings which originated from $\mathrm{sp}^{2}$ hybridized stretching vibrations of carbon bond. The presence of small 2D 
peaks further confirms that RGO sheets are highly crystalline in nature with lower defects confirming their high quality. ${ }^{38}$ The $I_{\mathrm{D}} / I_{\mathrm{G}}$ ratio measures the defects present in the carbon backbone of graphene sheets when dispersed and exfoliated in the presence of a fusion protein. ${ }^{39}$ The $I_{\mathrm{D}} / I_{\mathrm{G}}$ ratio for pristine RGO was found to be $\sim 0.35$, with the intensity of the D-band comparatively lower than that of the G-band, suggesting that the partial oxidization step did not introduce significant defects in the carbon backbone, as discussed earlier. The introduction of the fusion protein at different $\mathrm{pH}$ followed by the exfoliation of RGO sheets caused significant alterations in the $I_{\mathrm{D}} / I_{\mathrm{G}}$ values. Under conditions where the extended conformation of the RLP was anticipated (at $\mathrm{pH}>5$ ), the $I_{\mathrm{D}} / I_{\mathrm{G}}$ was found to be improved to $\sim 0.52$ and 0.42 at $\mathrm{pH} \sim 7$ and 11 , respectively. At $\mathrm{pH} \sim 4$, the fusion protein retains a random coil-like morphology and from $\frac{I_{\mathrm{D}}}{I_{\mathrm{G}}} \sim 0.37$ was found similar to pristine RGO sheets $(\sim 0.35) \cdot{ }^{40} \mathrm{It}$ showed that $I_{\mathrm{D}} / I_{\mathrm{G}}$ depended on the swellability of the $\mathrm{pH}$ responsive RLP linker connecting the RGO sheets. Similar observations of tuning the $\frac{I_{\mathrm{D}}}{I_{\mathrm{G}}}$ ratio were also reported in the case of molecularly engineered ionic liquids which were used for exfoliation of graphene sheets. ${ }^{41}$ This is due to the presence of specialized regions in the RLP consisting of alternate hydrophobic/hydrophilic patches which in the extended state improves the interfacial interaction with the RGO sheets. ${ }^{34}$ Along with it, HFBI is also known to specifically recognize and bind to the graphene surface through hydrophobic interactions. ${ }^{39}$ Under different $\mathrm{pH}$ conditions, the extendibility of hydrophilic/hydrophobic patches in the RLP varied due to which their interaction with RGO and the degree of exfoliation varied drastically. Sonication of RGO with a fusion protein resulted in different degrees of exfoliation at various $\mathrm{pH}$ which were sterically stabilized by NFC, as reported in our earlier study. ${ }^{42}$ Due to which the relative degree of structural defects introduced in RGO under different $\mathrm{pH}$ conditions varied which resulted in a tunable $I_{\mathrm{D}} / I_{\mathrm{G}}$ ratio. Therefore, extended conditions of the fusion protein (at $\mathrm{pH} \sim 7$ and 11) resulted in the introduction of a higher degree of structural defects in RGO (during sonication) due to their improved interaction compared to the random coiled state of the fusion protein (at $\mathrm{pH} \sim 4$ ). The above postulate was confirmed by investigating the $2 \mathrm{D}$ peak of NFC/ RGO processed at different $\mathrm{pH}$ (Fig. S4 $\dagger$ ), which shifted to a lower wavenumber (at $\mathrm{pH} \sim 7$ and 11) suggesting exfoliation or lowering in the number of stacked RGO sheets. ${ }^{38}$ To further examine the state of RGO dispersion in nanocomposites, the ratio of the intensity of the $2 \mathrm{D}$ band and $\mathrm{G}$ bands was investigated. It is well known that the $I_{2 \mathrm{D}} / I_{\mathrm{G}}$ ratios of single-, double-, triple-, and multi- $(>4)$ layer graphene sheets are typically $>1.6$, $\sim 0.8, \sim 0.30$, and $\sim 0.07$, respectively as per earlier reported studies. ${ }^{43,44}$ The $I_{2 \mathrm{D}} / I_{\mathrm{G}}$ is evaluated to be $\sim 0.25, \sim 0.29$ and $\sim 0.39$ for NFC/RGO films processed at $\mathrm{pH} \sim 4,7$ and 11 respectively. The results indicate that stacked RGO nanosheets consisting of less than $<3$ layers are intercalated with the fusion protein and NFC during nanocomposite formation, which was prevalent under all $\mathrm{pH}$ conditions. Furthermore, to confirm the number of layers of RGO sheets, the shape, components and intensity of the 2D peak were evaluated through detailed Raman spectroscopic analysis. In the case of Raman spectra for multilayered RGO, all vibrational modes split due to the confinement in the direction perpendicular to the basal plane and distinctive band structures are observed activated by the two-phonon DR Raman process (e.g., the 2D mode) which depends on the number of layers. ${ }^{38}$ From the Raman spectra, the 2D band for multilayered RGO is fitted with multiple Lorentzian peaks which correspond to multiple resonance electronic band structures of Raman processes. ${ }^{38}$ In the present study, the $2 \mathrm{D}$ peak has six components as deconvoluted by the Lorentzian fit (green curves), as shown in Fig. $\mathrm{S} 4, \dagger$ which is prevalent under all $\mathrm{pH}$ conditions for NFC/RGO films. The presence of six components in $2 \mathrm{D}$ peaks represents the presence of three layers of RGO sheets, which is similar to earlier reported studies ${ }^{38,45}$ and also in line with our earlier prediction with $I_{2 \mathrm{D}} / I_{\mathrm{G}}$ values. Furthermore, we measured the full width at half-maximum (FWHM) of the $2 \mathrm{D}$ band which is a quantitative guide to distinguish the layer number (single- to five-layer) in graphene sheets. In the present study, we observed that the FWHM of the 2D band for all the $\mathrm{NFC/RGO}$ films processed at different $\mathrm{pH}$ was in the range of $52-58 \mathrm{~cm}^{-1}$, which suggests the presence of tri-layered stacked RGO, as reported in earlier studies. ${ }^{46}$ Therefore, from Raman spectroscopic studies it can be confirmed that genetically engineered proteins can be strategically used to functionalize RGO/NFC through molecular recognition sites with effective dispersion and induction of $\mathrm{pH}$ responsive characteristics into fabricated nanocomposites.

In order to evaluate the effect of hybrid proteins on structural properties, a series of nanocomposites with variation in the protein content, $\mathrm{pH}$ and $\mathrm{RGO} / \mathrm{NFC}$ compositions were fabricated. The RGO/NFC films interestingly show a glassy appearance (as shown in ESI Fig. S1(b)†). The films appeared shiny with a mirror-like feature which showed a high reflection of the background surface (which was captured on the film as shown in Fig. S1(b) $\dagger$ ) and could be visualized by the naked eye. Similar observations of glassy thin films were reported by Mao et al. 2015 (ref. 16) analogous to nacre, which is due to the presence of the ultra-smooth surface and highly ordered lamellar structure of RGO and NFC. However, the appearance of high reflectivity suggests that such ordered structures were prevalent over longer ranges even on the macroscopic scale $(\sim 8-10 \mu \mathrm{m})$ of the films fabricated. The microscopic investigations of the surface and cross-sectional view of RGO/NFC nanocomposite films were performed by scanning electron microscopy (as shown in Fig. 2(b) and in ESI Fig. S2(a) $-(\mathrm{g}) \dagger)$. The electron micrographs from the side view of nanocomposites showed typical nacremimetic like structures with the presence of dense and alternate layers of RGO and NFC. From the top view, the films showed a highly smooth surface with the hierarchical structures predominant along the cross section without any defects (Fig. S2(a) and (b) †). The restacking of RGO sheets through $\pi-\pi$ electronic interactions and agglomeration of NFC due to hydrogen bonds were weakened through site-specific molecular recognition with HFBI and CBMs respectively. The highresolution micrographs show bundles of NFC nanofibers adsorbed onto the surface of RGO sheets forming a closely 
wrapped structure with high interfacial adhesion (Fig. S2(d) and (e) $\dagger$ ). The formation of highly ordered structures during evaporation-induced self-assembly with a 'brick-mortar pattern' is possibly mediated through molecular interactions from the fusion protein complex (evident from high-resolution SEM micrographs in Fig. 2(b) and (c)). On increasing the concentration of RGO as well as NFC, restacking of RGO sheets and agglomeration of cellulosic fibers were observed (Fig. S2(f) and $(\mathrm{g}) \dagger)$. However, it could be improved by tuning and optimizing the concentrations of the protein added during nanocomposite processing. Furthermore, to understand the degree of NFC/RGO packing in the interlayers we studied the film microstructure processed in different $\mathrm{pH}$ buffers (Fig. S3(a)-(c) †). At $\mathrm{pH} \sim 4$ and 7 , densely packed and compact layers of NFC/RGO were observed along the film depth with the RGO sheets aligned in a particular direction (evident from high-resolution micrographs in Fig. S3(a)†). The NFC_RGO_1_3_pH 7 films show a highly ordered and hierarchal architecture with stacked alternate layers of RGO and NFC, compared to films processed under other $\mathrm{pH}$ conditions. In sharp contrast, films processed at $\mathrm{pH} \sim 11$ were loosely packed with the presence of voids in between the NFC and RGO layers (Fig. S3(c) †). We measured the inter-layer spacing between the RGO and NFC sheets from FESEM micrographs which were $\sim 105 \mathrm{~nm}, 154 \mathrm{~nm}$ and $126 \mathrm{~nm}$ for the NFC/RGO films processed at $\mathrm{pH} 4,7$ and 10 respectively. Such a microstructural change is probably due to the expanded structure of resilin at higher $\mathrm{pH}$ or highly swollen cellulosic fibers under alkaline conditions. Therefore, it can be concluded that the micro-structures of NFC/RGO films could be tuned using functional fusion protein constructs which were expected to alter the mechanical and electrochemical behavior of the composites, as discussed in the subsequent section.

\section{Effect of the genetically engineered fusion protein on the mechanical behavior of nanocomposites}

To gain insight into the mechanical behavior of NFC/RGO nanocomposites processed with the fusion protein, tensile tests were carried out on several films with varying RGO content and dCBM-RLP-HFB concentration and different $\mathrm{pH}$ buffers as a medium for solvent casting (Fig. 3(a)-(c)). The NFC/RGO films processed without any addition of the fusion protein showed an ultimate tensile strength (UTS) of $\sim 40 \mathrm{MPa}$ and $\sim 24 \mathrm{MPa}$ at $\mathrm{pH}$ $\sim 7$ and at $\mathrm{pH} \sim 11$ respectively (Fig. S4(a) and (b) †). The change in the tensile modulus with $\mathrm{pH}$ can be attributed to the swelling of cellulose chains under alkaline conditions, which possibly increases the elongation behavior of the nanocomposites. ${ }^{47} \mathrm{On}$ introduction of the genetically engineered fusion protein, the mechanical behavior of nanocomposites showed a substantial improvement. For instance, the effect of the fusion protein concentration on the toughness and UTS was firstly investigated keeping the NFC/RGO content constant $(1: 3)$ at $\mathrm{pH} \sim 7$ (Fig. 3(b)). At a dCBM-RLP-HFB concentration of $\sim 2.2 \mathrm{~g} \mathrm{~L}^{-1}$, the UTS increased to $\sim 401 \mathrm{MPa}$ and toughness to $\sim 1.2 \mathrm{MJ} \mathrm{m}^{-3}$. It was observed that the theoretical maximal amount of bound fusion protein calculated from the binding isotherm plots needed for (NFC: RGO 1:3) is in line with the experimental values. Therefore, protein concentration added to the nanocomposite could be optimized using the binding isotherm plots studied in the earlier section, taking into consideration the content of RGO as a limiting factor. Secondly, the effect of different NFC : RGO ratios was studied keeping the content of the fusion protein constant at $\sim 1.5 \mathrm{~g} \mathrm{~L}^{-1}$ at $\mathrm{pH} \sim 7$. At a NFC : RGO of $1: 2$, the UTS was improved to $\sim 421 \mathrm{MPa}$ and for a NFC : RGO of $1: 3$ the toughness enhanced to $\sim 3.8 \mathrm{MJ}$ $\mathrm{m}^{-3}$ with a significant improvement in elongation at break. The simultaneous improvement in both the tensile modulus and toughness for the biomimetic nacre-based composites is seldom reported. ${ }^{1}$ The prominent advantage of the fusion protein complex is the presence of the supramolecular functional linker RLP which induces a pH-responsive behavior in the developed nanocomposites. Finally, the evaluation of different $\mathrm{pH}$ buffers as solvents for processing the NFC/RGO films keeping the protein concentration constant at $\sim 1.5 \mathrm{~g}$ $\mathrm{L}^{-1}$ and NFC : RGO at $1: 2$ was performed. At $\mathrm{pH} \sim 7$, the UTS increased to $\sim 421 \mathrm{MPa}$ but with a comparatively lower toughness of $\sim 3.02 \mathrm{MJ} \mathrm{m}^{-3}$, due to poor elongation behavior. Interestingly, it was observed that the fabricated nanocomposites showed $\mathrm{pH}$-dependent mechanical behavior because the multidomain dCBM-RLP-HFBI undergoes different structural changes under various $\mathrm{pH}$ conditions and ionization states.

A summary of the results of mechanical property investigations is presented in Fig. S5(a), $\uparrow$ to understand the effect of $\mathrm{pH}$, the ratio of NFC/RGO concentration and the content of the fusion protein on the UTS and toughness of the fabricated nanocomposites. The toughness and tensile strength varied from $\sim 0.8-3.84 \mathrm{MJ} \mathrm{m}^{-3}$ and $\sim 200-421 \mathrm{MPa}$ depending on the conformational state of the RLP under different conditions. Most of the reported GO-based nacre materials (as shown in Fig. S5(b) $\dagger$ ) such as GO-PVA, ${ }^{48}$ GO-PMMA, ${ }^{49}$ rGO-CNC, ${ }^{50}$ GODWNT-PCDO $,{ }^{51} \mathrm{GO}-\mathrm{Mg}^{2+},{ }^{22} \mathrm{GO}-\mathrm{CMC}-\mathrm{Mn}^{2+},{ }^{33} \mathrm{GO}-\mathrm{Zn},{ }^{54} \mathrm{rGO}-\mathrm{CS},{ }^{18}$ rGO-MMT-PVA, ${ }^{55}$ rGO-PAPB $^{56}$ and rGO-PDA $^{17}$ utilize either interfacial or synergistic interactions based on hydrogen bonding, hydrophobic/hydrophilic interactions, $\pi-\pi$ electronic interactions, and ionic and covalent bonding. From our previously reported study, ${ }^{21}$ the utilization of diblock proteins, dCBM-HFBI, in the fabrication of graphene/NFC films showed an improved tensile strength of $\sim 278 \mathrm{MPa}$ and toughness of $\sim 4.0 \mathrm{MJ} \mathrm{m}^{-3}$. The introduction of the functional unit RLP contributes to the fabrication of biomimetic-nacre with a larger tensile strength ( $\sim 21 \mathrm{MPa})$ imbued with stimuli-responsive characteristics. A typical fracture model is proposed to understand the reinforcing and strengthening mechanism of the fusion protein at different $\mathrm{pH}$ (as shown in Fig. 4). At $\mathrm{pH} \sim 4$, the RLP connector adopts a highly twisted and coiled-like structure which resists easy slippage between NFC and RGO layers, during the initial stages of stretching under tension. On increasing the $\mathrm{pH}$ to $\sim 7$, the RLP uncoils itself to adopt a freely extended conformation which gets gradually stretched under a tensile load. In this case, the assembled resilin-protein in between RGO and NFC layers acts as an elastic interface, which undergoes structural transitions under the tensile load. ${ }^{57}$ The molecular motifs, CBMs and HFBI adhere strongly to NFC and RGO creating an effective path for slippage in between the 


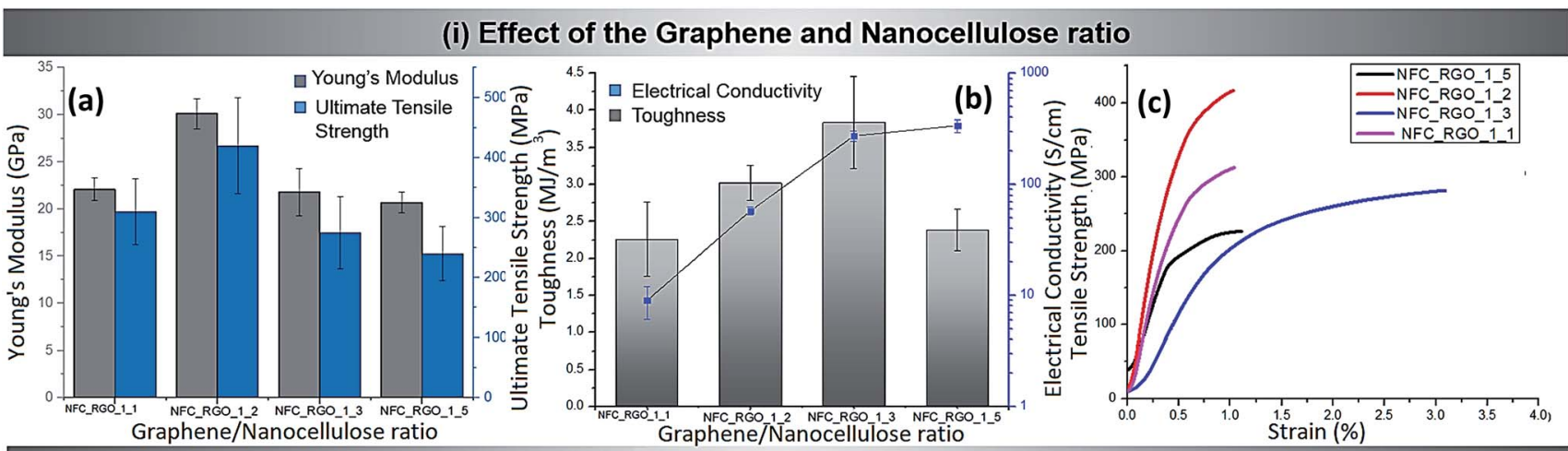

(ii) Effect of the dCBM-RLP-HFB concentration
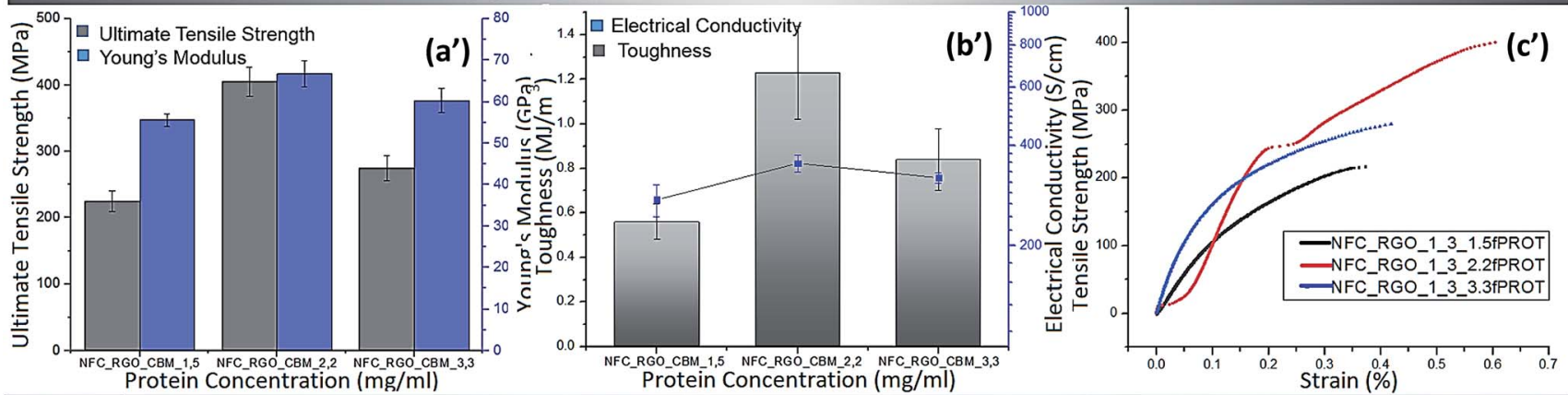

(iii) Effect of the different $\mathrm{pH}$
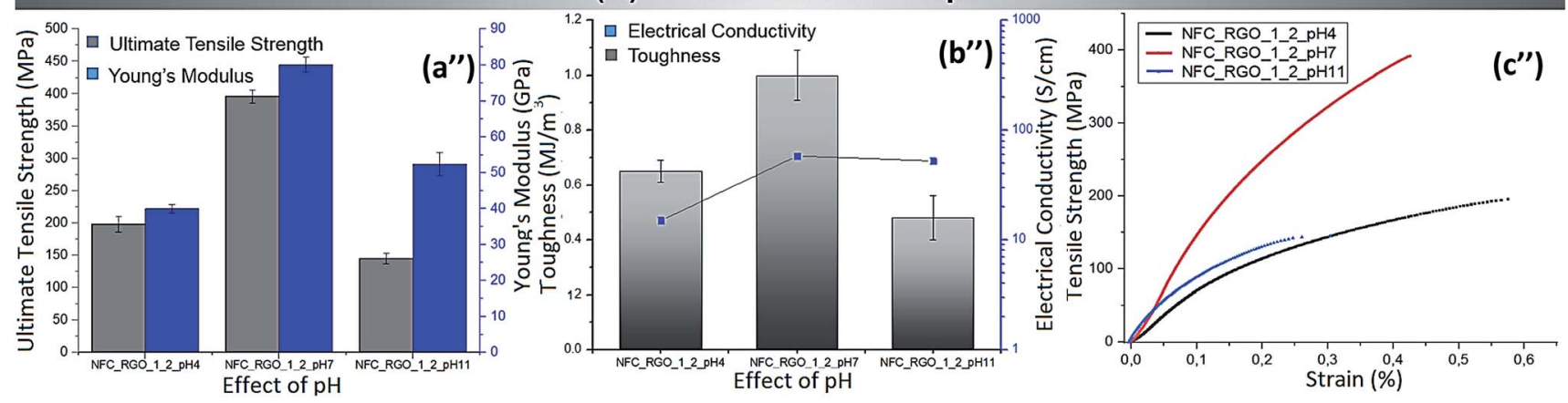

Fig. 3 Mechanical properties of the nanocomposite films containing (i) different ratios of NFC/RGO. (a) Effect on the ultimate tensile strength (UTS) and Young's modulus and (b) toughness and electrical conductivity of the nanocomposite films in the presence of various RGO/NFC ratios (with a constant protein concentration of $1.5 \mathrm{~g} \mathrm{~L}^{-1}$ at $\mathrm{pH}$ 7). (c) Stress-strain curves of the RGO/NFC films containing various concentrations of RGO. (ii) Effect of various concentrations of protein dCBM-RLP-HFB (at a constant NFC : RGO of $1: 3$ and pH 7) on the mechanical properties: $\left(a^{\prime}\right)$ UTS and YM and $\left(b^{\prime}\right)$ toughness and electrical conductivity, and $\left(c^{\prime}\right)$ typical stress-strain curves showing the effect of protein concentration. (iii) Effect of different $\mathrm{pH}$ conditions 4, 7 and 11 on the mechanical properties of RGO/NFC films: ( $\left.a^{\prime \prime}\right)$ UTS and YM and ( $\left.b^{\prime \prime}\right)$ toughness and electrical conductivity of NFC/RGO films at a ratio of $1: 2$ and a constant protein concentration of $1.5 \mathrm{~g} \mathrm{~L}^{-1}$ and ( $\mathrm{c}^{\prime \prime}$ ) stress-strain curve of NFC/RGO films when processed under different $\mathrm{pH}$ conditions.

adjacent layers, as it is gradually stretched. However, at $\mathrm{pH} \sim$ 11 , even though the fusion protein adopts the most extended state, cellulose undergoes increased swelling which possibly lowers the binding efficiency of molecular motifs with NFC/ RGO. From the molecular point of view, site-specific binding domains resulted in the effective transfer of stress between NFC and RGO and the flexible resilin segments improved toughness through synergistic interactions. It can be summarized that genetically engineered fusion proteins can significantly tune the structural properties of nanocomposites depending on the number of binding sites and the structural state of resilin protein, which can be governed by varying the conditions (such as $\mathrm{pH}$ and ionic strength) during processing.
Effect of the genetically engineered fusion protein on the electrochemical performance of films

Next, we wanted to study whether the modulation of the microstructures of nanocomposites influences the electrical properties, due to the responsive nature of the fusion protein. Such an altered hierarchical architecture can be controlled with $\mathrm{pH}$ and depends on the concentration of the fusion protein and RGO/ NFC ratio. The conductivity values measured were sufficiently high for all the nanocomposites, demonstrating a synergistic behavior similar to that of mechanical studies. The best value for the electrical conductivity was obtained for a NFC : RGO ratio of $1: 3$ with $2.2 \mathrm{~g} \mathrm{~L}^{-1}$ of dCBM-RLP-HFBI processed at $\mathrm{pH}$ 7 with a conductivity value of $\sim 680 \mathrm{~S} \mathrm{~cm}^{-1}$, suitable enough for 

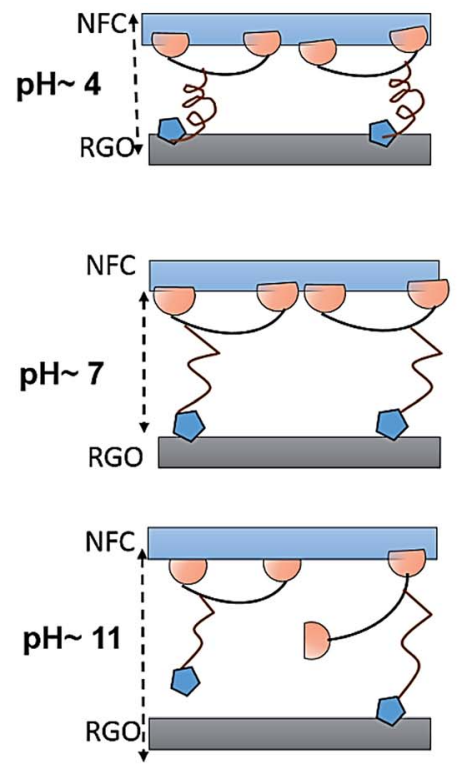

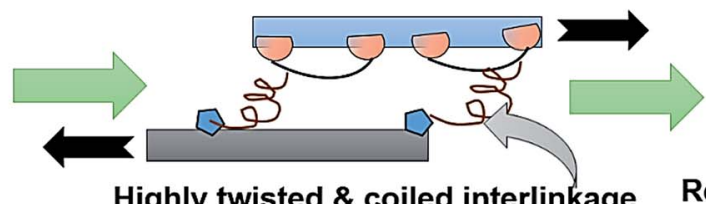

Highly twisted \& coiled interlinkage

\section{Restriction cause breaking of linkages}

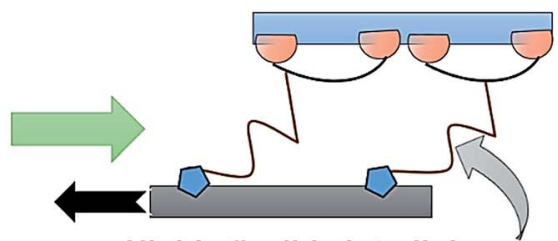

Highly flexible interlinkage

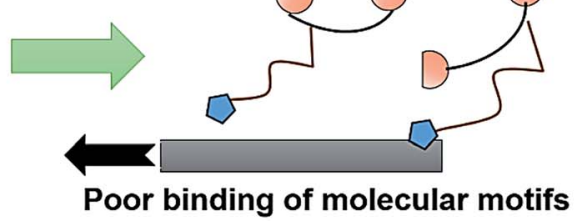

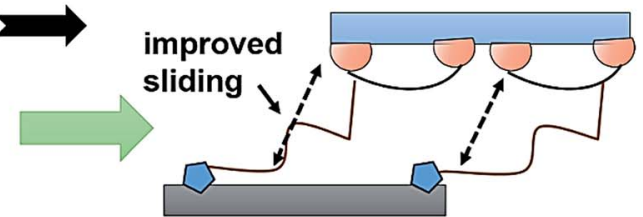

Swift slippage \& extensionability btw. layers

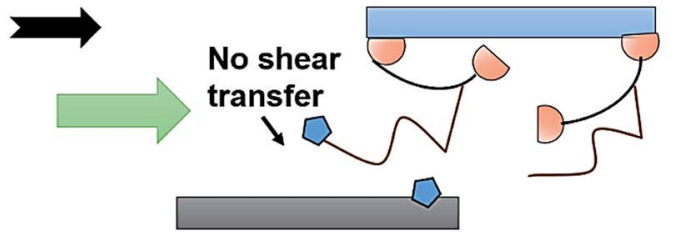

Low bonding \& rupturing of linkages

Fig. 4 Proposed fracture mechanism of RGO/NFC based nacre containing the fusion protein dCBM-RLP-HFBI, under different pH conditions (black arrows show the direction of the applied tensile stress).

most practical applications. As expected the electrical conductivity of NFC/RGO films increased with higher loadings of the RGO content and varied significantly with the change in the $\mathrm{pH}$ and concentration of the protein as detailed in Fig. $3(\mathrm{~b}),\left(\mathrm{b}^{\prime}\right)$ and $\left(b^{\prime \prime}\right)$. The electrical conductivity values almost reached saturation (at a NFC : RGO ratio of $1: 3$ at $\mathrm{pH} \sim 7$ ) and did not show any significant changes when the fusion protein or RGO concentration was increased. The functional units, RLPs, acted as bridges to connect the 'brick and mortar' architecture of densely packed adjacent layers of NFC/RGO (by improving interfacial interactions). The high electrical conductivity in combination with the mechanical robustness of NFC/RGO films has potential applications in flexible electronics. Moreover, the improved biocompatibility and $\mathrm{pH}$-responsive characteristics of the NFC/RGO films can be used for the fabrication of implantable bioelectrochemical capacitors. ${ }^{58}$ Taking into consideration the above two aspects, a study evaluating the electrochemical performance of NFC/RGO films infused with a $\mathrm{pH}$-responsive functional protein was performed, as a proof of concept. NFC/ RGO films at different loadings were fabricated to determine charge storage capabilities through evaluation of cyclic voltammograms (CV) and charge-discharge (CD) curves to optimize the ideal RGO content (as summarized in Fig. S7(a) and (b) †). The disparity in CV and CD curves (with the RGO content) is possibly due to aggregation and variation in the binding of fusion proteins with RGO/NFC, as already discussed in earlier sections. As confirmed in Fig. S7(a), $\dagger$ NFC_RGO_1_3 films due to their high electrochemical and mechanical stability were further processed at different $\mathrm{pH}$ to understand the effect of the functional protein on the electrochemical performance. Fig. 5(a) shows CV plots for NFC/RGO films (at $\mathrm{pH} \sim 4,7$ and 11) assembled into a three-electrode system using aqueous $6 \mathrm{M}$ $\mathrm{KOH}$ as the electrolyte. It should be noted that $\mathrm{KOH}$ as an electrolyte is widely used due to its small ionic radius $(r) \sim 2.92$ $\AA$ under hydrated condition ${ }^{59}$ and the ability to intercalate layers contributing to pseudo-capacitance. From XRD studies, the $d$-spacing for NFC/RGO films $(1: 3)$ was found to be in a similar range of $\sim 3.29,3.70$ and $3.42 \AA$, which can be tuned depending on $\mathrm{pH} \sim 4,7$ and 11 respectively. $\mathrm{CV}$ curves show a quasi-rectangle shape but with the presence of flatly roundish redox peaks in the $\mathrm{CV}$ curves which correspond to faradaic reactions due to the presence of defects and oxygen chemical functionalities in RGO. They are possibly introduced during partial oxidation of RGO, which are known to be active sites for faradaic redox reactions during the CV scans. A similar observation has also been observed in several other reported studies for reduced graphene oxide based materials. ${ }^{60-63}$ The significant difference in the integral area of the CV curves suggests variation in the charge storage capabilities of NFC/RGO films processed at different pH. At pH 7, NFC_RGO_1_3 films show the formation of a rectangular capacitive curve with the highest integral area which gradually decreases at $\mathrm{pH} \sim 11$ but undergoes distortion at $\mathrm{pH} \sim 4$. In NFC-RGO_1_3_pH 7, $d$ spacing higher than or closer to that of hydrated ions $(r<3.70 \AA)$ makes it readily accessible to the inter-layer galleries, creating a path for efficient ion transport. The composite contains sparsely filled NFC/RGO galleries, in which the ions can penetrate in inter-layers, resulting in overall higher charge storage capabilities. ${ }^{64}$ On the other hand, $d$-spacing at $\mathrm{pH} \sim 4$ and 11 was relatively smaller than or comparable to that of hydrated ions $(r \sim 3.29 \AA)$, which leads to ion sieving ${ }^{65}$ as they encounter increased resistance from RGO inter-layers. In this case, ion sorption mostly occurs on the surface of RGO layers due to the resistance provided by small pores and densely filled galleries of NFC/RGO. Furthermore, evaluation of $\mathrm{CV}$ curves for NFC_RGO_1_3_pH 7 at higher scan rates (from $\sim 10$ to $100 \mathrm{mV}$ 

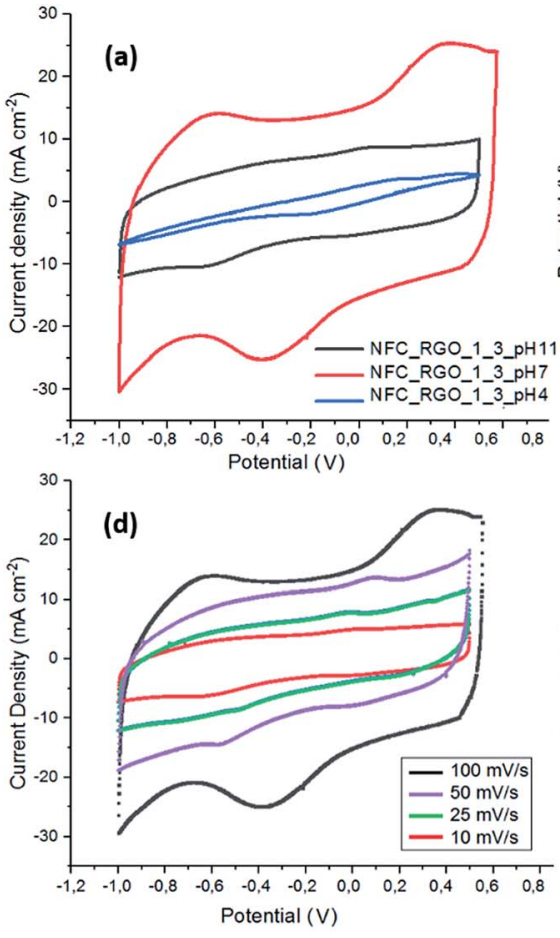
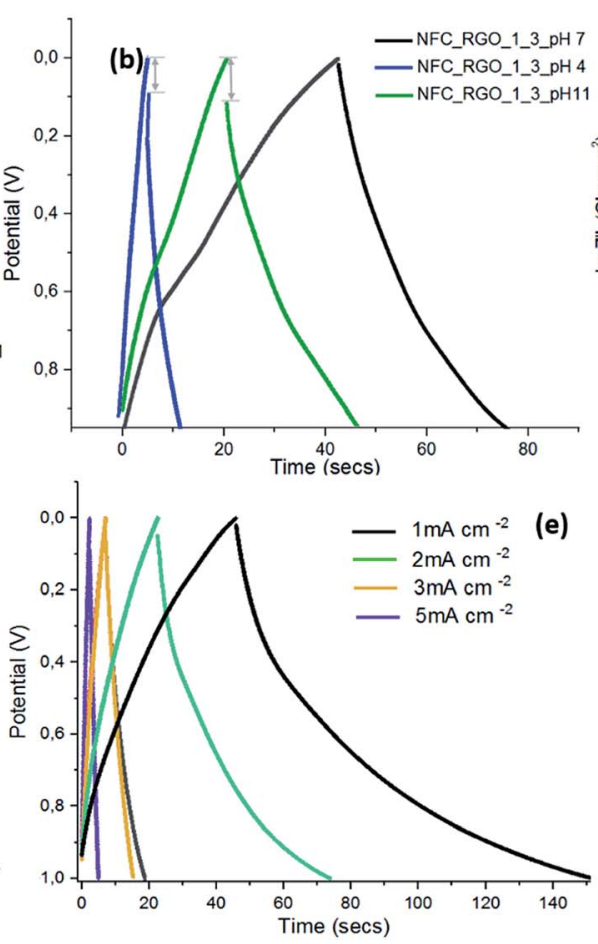
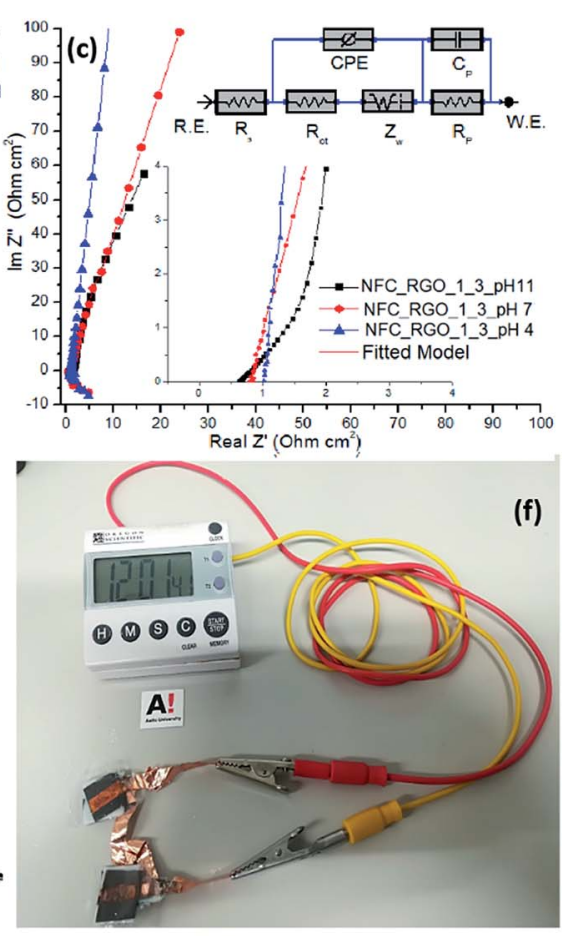

Fig. 5 Electrochemical performance of the fabricated free-standing NFC/RGO films measured in a three-electrode system: (a) CV curves of $\mathrm{NFC/RGO}$ films at a $1: 3$ ratio processed with $\sim 2.2 \mathrm{~g} \mathrm{~L}^{-1} \mathrm{CBM}$ under three different $\mathrm{pH}$ conditions ( $\mathrm{pH}=4,7$ and 11 ), (b) galvanostatic chargedischarge curves of the RGO/NFC films at a current density of $2 \mathrm{~mA} \mathrm{~cm}^{-2}$, (c) Nyquist plots of RGO/NFC films with the inset showing an enlarged view of the high frequency region and the equivalent electrical circuit used for data fitting, (d) CV curve of the RGO/NFC films at a $1: 3$ ratio processed at $\mathrm{pH} 7$ and measured at different scan rates $\left(\mathrm{mV} \mathrm{s}^{-1}\right)$, (e) galvanostatic charge-discharge curve of the same films measured at different current densities, and (f) two flexible solid-state super-capacitors made from the genetically engineered protein-infused free-standing $\mathrm{RGO/NFC} \mathrm{films} \mathrm{which} \mathrm{are} \mathrm{connected} \mathrm{in} \mathrm{series} \mathrm{to} \mathrm{illuminate} \mathrm{a} \mathrm{LED} \mathrm{circuit} \mathrm{present} \mathrm{in} \mathrm{a} \mathrm{household} \mathrm{electronic} \mathrm{watch} \mathrm{of} 1.5 \mathrm{~V}$.

$\mathrm{s}^{-1}$ ) (as shown in Fig. 5(d)) still shows the quasi-rectangular nature with the presence of redox peaks in a wide potential window of $-1 \mathrm{~V}$ to $0.5 \mathrm{~V}$. This suggests that the fabricated NFC/ RGO films show a hybrid electrochemical behavior with the presence of pseudocapacitance as well as electrochemical double layer (EDL) capacitive behavior which have improved stability and potentially higher rate retention capabilities.

Electrochemical impedance spectroscopy (EIS) analysis was carried out to further understand the ion-diffusion and transport mechanism in the $\mathrm{pH}$ tunable interlayer spacing of $\mathrm{NFC} /$ RGO films. The Nyquist plot shows a vertical line almost parallel to the $y$-axis at lower frequencies which represents the pure capacitive behavior of NFC/RGO films (Fig. 5(c)). The absence of the semi-circle along with the intermediate $45^{\circ}$ region is observed for all the films processed with an optimized fusion protein content of $\sim 2.2 \mathrm{~g} \mathrm{~L}^{-1}$. This suggests that the internal resistance of current collectors arising from electrode contacts and electrolyte interference was negligible. ${ }^{66}$ The measured equivalent series resistance (ESR) from the Nyquist plots (intercept with the $Z^{\prime}$ axis at lower frequency) was found to be $\sim 1.01,0.832$ and $0.628 \Omega . \mathrm{cm}^{2}$ for the films processed at $\mathrm{pH} \sim$ 4, 7 and 11 respectively. The equivalent resistance values are exceptionally small (much lower than those in previously reported studies as shown in Table $\mathrm{S} 2 \dagger$ ) and are reduced by half in comparison to the ultra-tough and highly conductive $\mathrm{rGO} / \mathrm{CNC}$ composites, recently reported in the literature (with values of $\left.\sim 1.48 \Omega \mathrm{cm}^{2}\right) .{ }^{50}$ The fusion protein with selective molecular binding motifs to NFC and RGO provides an excellent interfacial contact resulting in improved ion transport as well as the fast transfer of electrons. Furthermore, the EIS experimental data were fitted with an equivalent circuit diagram (as shown in Fig. 5(c)) which comprised the following elements: (i) solution resistance $\left(R_{\mathrm{S}}\right)$, which takes into account the inherent resistance of the active electrode and contact resistance arising from the electrodes, current collectors and electrolyte, ${ }^{67}$ (ii) charge transfer resistance $\left(R_{\mathrm{ct}}\right)$, which arises at the surface of the electrode and electrolyte interface, ${ }^{68}$ (iii) constant phase element (CPE), which is a double layer capacitance that occurs due to the porous or hierarchal structure of NFC/RGO layers at the interface with electrolytes due to charge separation, and (iv) Warburg diffusion resistance $(W)$ which represents the ion penetration phenomenon across the NFC/RGO inter-layers. The pseudocapacitance $\left(C_{\mathrm{p}}\right)$ and charge separation resistance $\left(R_{\mathrm{p}}\right)$ connected in a parallel circuit refer to the ideal capacitive behavior in which the Nyquist plot ideally forms a straight line parallel to the $y$-axis. ${ }^{69}$ The fitting curves for the Nyquist plots coincide well with the experimental values (with less than 5\% error), suggesting that the proposed equivalent circuit model will reflect the actual electrochemical processes occurring within the hybrid protein based NFC/RGO supercapacitors. The 
circuit parameters obtained after fitting the equivalent circuit model are mentioned in Table S2. $\dagger$ Based on the fitting result of the equivalent circuit, the estimated values of $R_{\mathrm{s}}$ are 1.08, 0.78 and $0.64 \Omega$ for NFC/RGO films processed at $\mathrm{pH} \sim 4,7$ and 11 respectively, which are in line with the ESR values (as discussed earlier). The estimated values of $R_{\mathrm{ct}}$ are 1.54, 1.24 and $1.26 \Omega$ for NFC/RGO films processed under $\mathrm{pH}$ conditions $\sim 4,7$ and 11 respectively, which are relatively low in magnitude. This is probably because of the presence of a well-connected interface between NFC/RGO layers induced by the site-specific binding units of hybrid proteins which is expected to lower the faradaic resistance. The absence of the semi-circular region in the Nyquist plots further confirms that the charge storage is governed by a non-faradaic process, a desirable feature in supercapacitors. $^{70}$ The Nyquist plot at medium frequency shows a slope of $45^{\circ}$, which can be represented by the Warburg resistance in the equivalent circuit. ${ }^{71}$ It was observed that the projected length of the Warburg region in Nyquist plots was shorter for NFC/RGO films processed at $\mathrm{pH} \sim 4$ and 7 in comparison to that at $\mathrm{pH} \sim 11$. NFC_RGO_1_3_pH 11 shows a slight shift to $45^{\circ}$ (in the medium frequency range, inset Fig. 5(c)) compared to others suggesting resistance to ion transport due to the formation of a diffuse electrical double layer. The Warburg coefficient measured was $134.8 \times 10^{-6}, 9.4$ $\times 10^{-6}$ and $76.3 \times 10^{-6} \Omega \mathrm{s}^{-1 / 2}$ for the NFC/RGO films processed under $\mathrm{pH}$ conditions $\sim 4,7$ and 11 respectively. Such variations are probably due to a higher $d$-spacing of $\sim 3.70 \AA$ at $\mathrm{pH} \sim 7$ (as confirmed from XRD) which provides fast ion diffusion across hierarchical NFC/RGO inter-layers (as shown in FESEM micrographs, Fig. 2(c)), resulting in easy accessibility of aqueous electrolytes to RGO nanosheets. Therefore, it can be observed that increased transport of ions results in improved rate capabilities and lowered resistance due to shorter diffusion paths, which can be explicitly tuned by varying the $\mathrm{pH}$ responsive functional linker. The CPE element, $Y_{\mathrm{o}}$, details the deviations observed in ideal capacitive properties due to the presence of non-uniform reaction sites, physical defects and charge transfer resistance at electrodes. The low $Y_{\mathrm{o}}$ values for NFC/RGO films with hybrid proteins especially processed at $\mathrm{pH}$ $\sim 7$ suggest an improved interfacial contact and the absence of physical defects. Furthermore, the evaluation of the power factor $n$ determines the degree of ideal capacitive properties of electrodes, which is generally in range of $0.5<n<1$, for supercapacitors. The measured $n$ values for NFC/RGO films processed at different $\mathrm{pH}$ were in the range of $0.82-0.98$, which are closer to $\sim 1$ suggesting ideal capacitive behavior, which is in line with earlier reported studies. ${ }^{72}$ Among all NFC/RGO films processed under different pH conditions, NFC-RGO_1_3_pH 7 based solid state films have improved electronic and ionic transport capabilities, which have been further investigated.

The evaluation of real $\left(C^{\prime}(\omega)\right)$ and imaginary capacitance $\left(C^{\prime \prime}(\omega)\right)$, a frequency-dependent term, provides insights into stored energy and losses due to ionic resistance arising from the microstructure of the NFC/RGO films. In $C^{\prime \prime}(\omega) v s$. frequency plots, the NFC/RGO films display a time constant $\tau_{\text {o }}$ of $\sim 0.80$, 0.59 and $0.88 \mathrm{~s}$ for the films processed at $\mathrm{pH} \sim 4,7$ and 11 respectively (Fig. S8(a) $\dagger$ ). The lowered $\tau_{\mathrm{o}}$ value for NFC-
RGO_1_3_pH 7 suggests improved transition from resistive to capacitive behavior. ${ }^{64}$ The increased inter-layer spacing and lower resistance at $\mathrm{pH} \sim 7$ facilitate the effective transport of solvated $\mathrm{K}^{+}$and $\mathrm{HO}^{-}$ions, leading to a pseudocapacitance-like behavior. Interestingly, the real $C^{\prime}(\omega)$ component which measures the ability to store energy for NFC/RGO films varied significantly with $\mathrm{pH}$ even though the concentrations of NFC/ RGO and the protein remained constant. The maximum for $C^{\prime}(\omega)$ was nearly three times higher for NFC/RGO films processed at $\mathrm{pH} \sim 7$ in comparison to those at $\mathrm{pH} \sim 4$ and 11. It could be attributed to increased ion adsorption at the surface as well as inter-galleries and negligible resistance provided to ion transport in NFC-RGO_1_3_pH 7 films. ${ }^{73}$ However, at pH $\sim 4$ and 11 lower $d$-spacing resisted the access of solvated ions to the galleries of NFC/RGO, leading to lowered capacitive behavior. Fig. $\mathrm{S} 9 \dagger$ schematically summarizes the physicochemical ion transport mechanism and dynamics prevailing in NFC/RGO films when processed under different $\mathrm{pH}$ conditions. Two main parameters, firstly improved ion transport and sorption in the inter-spacing of RGO (lowered $\tau_{\mathrm{o}}$ ) and negligible resistance at the electrode/electrolyte interface (low $Z^{\prime \prime}$ ), contributed to improved capacitive behavior at $\mathrm{pH} \sim 7$. The difference in capacitive properties highlights the importance of fusion proteins, which have the capability to fine-tune material microstructures and hence require optimization to exactly match pore-size with that of the selected electrolyte ions for study.

The conclusions derived from the EIS analysis were validated with galvanostatic charge-discharge (CD) studies to determine the charge storage capacity of NFC/RGO protein-based supercapacitors. Fig. 5(b) displays a typical quasi-triangular CD curve with a significant difference in discharge times for NFC/RGO films processed under various $\mathrm{pH}$ conditions. The measured specific areal capacitance was found to be $\sim 20.8,71.2$ and 52.1 $\mathrm{mF} \mathrm{cm}{ }^{-2}$ at $\mathrm{pH} \sim 4,7$ and 11 respectively measured at a current density of $\sim 2 \mathrm{~mA} \mathrm{~cm} \mathrm{~cm}^{-2}$ and volumetric capacitance was measured to be $\sim 26,89$ and $65.1 \mathrm{~F} \mathrm{~cm}^{-3}$ at $\mathrm{pH} \sim 4,7$ and 11 respectively at similar current density. Furthermore, the $\mathrm{CD}$ curves for NFC-RGO_1_3_pH 7 films at various current densities (from 1-5 $\mathrm{mA} \mathrm{cm}^{-2}$ ) (Fig. 5(e)) showed high stability and symmetricity without any significant voltage (or IR) drop. This suggests the presence of improved mass transport of ions across the RGO layers with the formation of a stable EDL even at higher current densities, as already discussed in the earlier section. However, the evaluation of the CD curves at different ratios of NFC/RGO showed the presence of a slight IR drop in the range of 0.04-0.107 (Fig. S7(b) †). The drop is basically due to agglomeration of NFC/RGO flakes in the presence of lower or higher fractions of the fusion protein. Therefore, it is significantly important to determine the optimized protein concentration from the binding isotherms for the fabrication of NFC/ RGO films with ideal electrochemical performance. To further evaluate the practical applications of such protein-based NFC/ RGO films in bioelectronic devices, rate and cycling performance tests were carried out. The cycling stability of NFC/RGO films was measured at a high current density of $25 \mathrm{~mA} \mathrm{~cm}^{-2}$ to determine the percentage capacitance retention which showed a drop by $\sim 60.1,31.3$ and $25.7 \%$ for the films processed at $\mathrm{pH} \sim$ 
4, 7 and 11 respectively. The significant drop was probably due to the poor stability of the fusion protein, dCBM-RLP-HFBI, at high current density under alkaline conditions during the cycling tests which last for longer time scales. A similar behavior of high voltage loss was also observed for tests conducted on larger time scales in the case of earlier reported study of ultrathin graphene-protein (bovine serum albumin or silk) based supercapacitors. ${ }^{58,74}$ On reducing the current density to $1 \mathrm{~mA}$ $\mathrm{cm}^{-2}$ for cycling stability tests, the NFC_RGO_1_3_pH 7 films show higher capacitance retention with a small drop of $\sim 11.2 \%$ observed after 1000 cycles. However, potential applications of the developed protein-based supercapacitors as implantable biomedical devices require combined high mechanical and electrochemical stability under harsh biological environments.

In the earlier section, we have demonstrated the high mechanical integrity of NFC/RGO films intercalated with the fusion protein complex, resulting in the formation of tough composites comparable to biological materials such as nacre. Now to determine the electrochemical stability of the films, CV tests were measured under different mechanical stress and bending cycles (wrapped over a cylinder of diameter $\sim 1 \mathrm{~cm}$ as shown in Fig. S8(e) and (f)†). The highly flexible NFC/RGO films did not show any significant changes in the CV plots with the presence of a quasi-rectangular shape, under applied mechanical bending stresses. To demonstrate the hands-on application of the fabricated NFC/RGO films modified with the genetically engineered and functional protein complex, two free-standing electrodes were connected in series to illuminate an electronic watch, a house-hold daily appliance (shown in Fig. 5(f)). Furthermore, the capacitive and the mechanical performance of the fabricated NFC/RGO films were compared with those of cellulose hybrid composites reported in the literature as shown in Table S3. $\dagger$ From previously reported studies, NFC/RGO films composed of hybrid proteins have significantly high electrochemical performance and extremely low resistance along with superior mechanical properties, suitable enough for potential engineering applications. Interestingly, the engineered protein provided us sole functionality to remotely tune the microstructure of RGO/NFC which could be used as a tool to match the ionic radius of electrolytes used, to maximize the supercapacitive behavior of developed composites. Therefore, selective designing of genetically engineered proteins provides a unique approach to fabricate functional nanocomposites with high electrochemical stability and mechanical robustness, suitable for flexible and implantable biomedical devices.

\section{Conclusions}

This work demonstrates that genetic engineering of proteins at molecular levels could effectively transform their functionality with the currently developed dCBM-RLP-HFBI, acting as an elastic and energy dissipative interface in hybrid nanocomposites. The efficacy of the present study depends on the interaction of fusion proteins with NFC and RGO, which follows a cooperative binding behavior over multiple sites. The fundamental challenges associated with traditional graphene and nanocellulose related composites are poor interfacial interactions and material contacts. In this study, the traditional problems have been overcome using genetically engineered fusion proteins which act as bridges interconnecting the two layers strongly through site-specific molecular recognition, preventing their restacking. The evaluation of the microstructure showed that the surface-bound hybrid protein acted as a spacer tuning the $d$-spacing of inter-galleries depending on $\mathrm{pH}$ for processing of the films. This led to a significant effect on the degree of intercalation of NFC and altered the selective adsorption of electrolyte ions which caused pronounced changes to structural as well as electrochemical behavior. The highly stiff graphene and NFC were bonded strongly to diblock functional proteins, through material-specific binding motifs and resilin at the interface acts as a 'spring' transferring high resilience behavior to the composite. The underlying mechanism for simultaneous improvement of strength and toughness through synergistic interactions was investigated through fracture studies. The modular protein coupled with functional linkages underwent reversible conformational changes of swelling and contractions over changing $\mathrm{pH}$ conditions. The pH-responsive structural changes precisely governed the transport and diffusion of solvated electrolyte ions through NFC/RGO galleries. The present approach of tuning interspacing using functional proteins provided a unique way to optimize the electrochemical performance of supercapacitors depending on the solvated radius of electrolytes selected for the study. Interestingly, the current approach of molecular engineering of biomimetic nanocomposites caused no internal resistance arising from poor electrical or electrolyte contacts with the formation of a highly stable electrical double layer over longer cycles. Though obtaining cost-effective technologies for bioprocessing genetically engineered proteins remains challenging, their application as material specific binders which results in negligible internal resistance (at optimized fractions) provides promising engineering solutions for electronic appliances. The present study opens up new avenues for the production of hybrid materials using multifunctional bioengineered proteins for effective material contacts, which can be used as a replacement to metallic components present in microelectronics, with a novel prospect for the development of next-generation protein-based electrical devices.

\section{Conflicts of interest}

The authors declare no conflicts to declare.

\section{Acknowledgements}

The authors would like to thank the Nanomicroscopy Centre (NMC), Aalto University for the scanning electron microscopy (SEM) facility and Bart Rooijakkers for his help with ultra-high performance liquid chromatography (UHPLC) for protein analysis. Riitta Suihkonen is thanked for help in the protein purification. We are grateful for the support by the FinnCERES Materials Bioeconomy Ecosystem and Academy of Finland Centre of Excellence Programme (HYBER 2014-2019). 


\section{Notes and references}

1 Y. Zhang, S. Gong, Q. Zhang, P. Ming, S. Wan, J. Peng, L. Jiang and Q. Cheng, Chem. Soc. Rev., 2016, 45, 2378-2395.

2 S. Y. Bahn, B. H. Jo, Y. S. Choi and H. J. Cha, Sci. Adv., 2017, 3, e1700765.

3 G. Jain, M. Pendola, Y.-C. Huang, J. Juan Colas, D. Gebauer, S. Johnson and J. S. Evans, Biochemistry, 2017, 56, 3607-3618. 4 G. Qin, X. Hu, P. Cebe and D. L. Kaplan, Nat. Commun., 2012, 3, 1003.

5 C. M. Elvin, A. G. Carr, M. G. Huson, J. M. Maxwell, R. D. Pearson, T. Vuocolo, N. E. Liyou, D. C. C. Wong, D. J. Merritt and N. E. Dixon, Nature, 2005, 437, 999-1002.

6 R. Balu, R. Knott, N. P. Cowieson, C. M. Elvin, A. J. Hill, N. R. Choudhury and N. K. Dutta, Sci. Rep., 2015, 5, 10896.

7 A. Chilkoti, T. Christensen and J. A. MacKay, Curr. Opin. Chem. Biol., 2006, 10, 652-657.

8 C. Vepari and D. L. Kaplan, Prog. Polym. Sci., 2007, 32, 9911007.

9 R. Balu, J. Whittaker, N. K. Dutta, C. M. Elvin and N. R. Choudhury, J. Mater. Chem. B, 2014, 2, 5936-5947.

10 H. Deng, Y. Su, M. Hu, X. Jin, L. He, Y. Pang, R. Dong and X. Zhu, Macromolecules, 2015, 48, 5969-5979.

11 R. S.-C. Su, J. N. Renner and J. C. Liu, Biomacromolecules, 2013, 14, 4301-4308.

12 B. Henrissat, Cellulose, 1994, 1, 169-196.

13 M. B. Linder, Curr. Opin. Colloid Interface Sci., 2009, 14, 356363.

14 A. M. Belcher, X. H. Wu, R. J. Christensen, P. K. Hansma, G. D. Stucky and D. E. Morse, Nature, 1996, 381, 56.

15 M. Suzuki, K. Saruwatari, T. Kogure, Y. Yamamoto, T. Nishimura, T. Kato and H. Nagasawa, Science, 2009, 325, 1388-1390.

16 L.-B. Mao, H.-L. Gao, H.-B. Yao, L. Liu, H. Cölfen, G. Liu, S.-M. Chen, S.-K. Li, Y.-X. Yan, Y.-Y. Liu and S.-H. Yu, Science, 2016, 354, 107-110.

17 W. Cui, M. Li, J. Liu, B. Wang, C. Zhang, L. Jiang and Q. Cheng, ACS Nano, 2014, 8, 9511-9517.

18 S. Wan, J. Peng, Y. Li, H. Hu, L. Jiang and Q. Cheng, ACS Nano, 2015, 9, 9830-9836.

19 Y. Wang, T. Li, P. Ma, S. Zhang, H. Zhang, M. Du, Y. Xie, M. Chen, W. Dong and W. Ming, ACS Nano, 2018, 12, 6228-6235.

20 J.-M. Malho, S. Arola, P. Laaksonen, G. R. Szilvay, O. Ikkala and M. B. Linder, Angew. Chem., Int. Ed., 2015, 54, 1202512028.

21 P. Laaksonen, A. Walther, J.-M. Malho, M. Kainlauri, O. Ikkala and M. B. Linder, Angew. Chem., Int. Ed., 2011, 50, 8688-8691.

22 H. Rajabi, A. Shafiei, A. Darvizeh and S. N. Gorb, Sci. Rep., 2016, 6, 39039.

23 S. Arola and M. B. Linder, Sci. Rep., 2016, 6, 35358.

24 J. Lehtiö, J. Sugiyama, M. Gustavsson, L. Fransson, M. Linder and T. T. Teeri, Proc. Natl. Acad. Sci. U. S. A., 2003, 100, 484489.
25 G. R. Szilvay, A. Paananen, K. Laurikainen, E. Vuorimaa, H. Lemmetyinen, J. Peltonen and M. B. Linder, Biochemistry, 2007, 46, 2345-2354.

26 A. Griffo, H. Hähl, S. Grandthyll, F. Müller, A. Paananen, M. Ilmén, G. R. Szilvay, C. P. Landowski, M. Penttilä, K. Jacobs and P. Laaksonen, ACS Omega, 2017, 2, 6906-6915.

27 W. Fang, A. Paananen, M. Vitikainen, S. Koskela, A. Westerholm-Parvinen, J. J. Joensuu, C. P. Landowski, M. Penttilä, M. B. Linder and P. Laaksonen, Biomacromolecules, 2017, 18, 1866-1873.

28 A. Ladiwala, K. Rege, C. M. Breneman and S. M. Cramer, Proc. Natl. Acad. Sci. U. S. A., 2005, 102, 11710-11715.

29 A. W. Marczewski, Langmuir, 2010, 26, 15229-15238.

30 J. Haiech, Y. Gendrault, M.-C. Kilhoffer, R. Ranjeva, M. Madec and C. Lallement, Biochim. Biophys. Acta Mol. Cell Res., 2014, 1843, 2348-2355.

31 Kenry, K. P. Loh and C. T. Lim, Nanoscale, 2016, 8, 94259441.

32 D. I. Cattoni, O. Chara, S. B. Kaufman and F. L. G. Flecha, PLoS One, 2015, 10, e0146043.

33 A. A. Shemetov, I. Nabiev and A. Sukhanova, ACS Nano, 2012, 6, 4585-4602.

34 W. Fang, Nonappa, M. Vitikainen, P. Mohammadi, S. Koskela, M. Soikkeli, A. Westerholm-Parvinen, C. P. Landowski, M. Penttilä, M. B. Linder and P. Laaksonen, Colloids Surf., B, 2018, 171, 590-596.

35 P. Dhar, S. S. Gaur, A. Kumar and V. Katiyar, Sci. Rep., 2018, 8, 3886.

36 J.-B. Wu, M.-L. Lin, X. Cong, H.-N. Liu and P.-H. Tan, Chem. Soc. Rev., 2018, 47, 1822-1873.

37 A. C. Ferrari and D. M. Basko, Nat. Nanotechnol., 2013, 8, 235-246.

38 J.-B. Wu, M.-L. Lin, X. Cong, H.-N. Liu and P.-H. Tan, Chem. Soc. Rev., 2018, 47, 1822-1873.

39 P. Laaksonen, M. Kainlauri, T. Laaksonen, A. Shchepetov, H. Jiang, J. Ahopelto and M. B. Linder, Angew. Chem., Int. Ed., 2010, 49, 4946-4949.

40 S. Gayathri, P. Jayabal, M. Kottaisamy and V. Ramakrishnan, AIP Adv., 2014, 4, 027116.

41 M. Matsumoto, Y. Saito, C. Park, T. Fukushima and T. Aida, Nat. Chem., 2015, 7, 730-736.

42 P. Laaksonen, A. Walther, J.-M. Malho, M. Kainlauri, O. Ikkala and M. B. Linder, Angew. Chem., Int. Ed., 2011, 50, 8688-8691.

43 H. Luo, J. Dong, F. Yao, Z. Yang, W. Li, J. Wang, X. Xu, J. Hu and Y. Wan, Nano-Micro Lett., 2018, 10, 42.

44 H. Luo, J. Dong, F. Yao, Z. Yang, W. Li, J. Wang, X. Xu, J. Hu and Y. Wan, Nano-Micro Lett., 2018, 10, 42.

45 W. Zhao, P. Tan, J. Zhang and J. Liu, Phys. Rev. B: Condens. Matter Mater. Phys., 2010, 82, 245423.

46 Y. Hao, Y. Wang, L. Wang, Z. Ni, Z. Wang, R. Wang, C. K. Koo, Z. Shen and J. T. L. Thong, Small, 2010, 6, 195-200.

47 L. Li, L. Liu, Y. Qing, Z. Zhang, N. Yan, Y. Wu and C. Tian, Electrochim. Acta, 2018, 270, 302-309.

48 Y. Wang, H. Yuan, P. Ma, H. Bai, M. Chen, W. Dong, Y. Xie and Y. S. Deshmukh, ACS Appl. Mater. Interfaces, 2017, 9, 4215-4222. 
49 E. Paz, F. Forriol, J. C. del Real and N. Dunne, Mater. Sci. Eng. C, 2017, 77, 1003-1011.

50 Y. Wen, M. Wu, M. Zhang, C. Li and G. Shi, Adv. Mater., 2017, 29, 1702831.

51 S. Gong, W. Cui, Q. Zhang, A. Cao, L. Jiang and Q. Cheng, ACS Nano, 2015, 9, 11568-11573.

52 S. Park, K.-S. Lee, G. Bozoklu, W. Cai, S. T. Nguyen and R. S. Ruoff, ACS Nano, 2008, 2, 572-578.

53 K. Shahzadi, X. Zhang, I. Mohsin, X. Ge, Y. Jiang, H. Peng, H. Liu, H. Li and X. Mu, ACS Nano, 2017, 11, 5717-5725.

54 D. V. Lam, T. Gong, S. Won, J.-H. Kim, H.-J. Lee, C. Lee and S.-M. Lee, Chem. Commun., 2015, 51, 2671-2674.

55 P. Ming, Z. Song, S. Gong, Y. Zhang, J. Duan, Q. Zhang, L. Jiang and Q. Cheng, J. Mater. Chem. A, 2015, 3, 2119421200.

56 M. Zhang, L. Huang, J. Chen, C. Li and G. Shi, Adv. Mater., 2014, 26, 7588-7592.

57 R. Xiong, H. S. Kim, L. Zhang, V. F. Korolovych, S. Zhang, Y. G. Yingling and V. V. Tsukruk, Angew. Chem., 2018, 130, 8644-8649.

58 I. M. Mosa, A. Pattammattel, K. Kadimisetty, P. Pande, M. F. El-Kady, G. W. Bishop, M. Novak, R. B. Kaner, A. K. Basu, C. V. Kumar and J. F. Rusling, Adv. Energy Mater., 2017, 7, 1700358.

59 Y. Marcus, Chem. Rev., 1988, 88, 1475-1498.

60 F. Yang, L. Zhang, A. Zuzuarregui, K. Gregorczyk, L. Li, M. Beltrán, C. Tollan, J. Brede, C. Rogero, A. Chuvilin and M. Knez, ACS Appl. Mater. Interfaces, 2015, 7, 20513-20519.

61 Y. J. Oh, J. J. Yoo, Y. I. Kim, J. K. Yoon, H. N. Yoon, J.-H. Kim and S. B. Park, Electrochim. Acta, 2014, 116, 118-128.
62 D. P. Dubal, J. Suarez-Guevara, D. Tonti, E. Enciso and P. Gomez-Romero, J. Mater. Chem. A, 2015, 3, 23483-23492.

63 M. Jana, S. Saha, P. Khanra, P. Samanta, H. Koo, N. C. Murmu and T. Kuila, J. Mater. Chem. A, 2015, 3, 7323-7331.

64 H. Banda, S. Périé, B. Daffos, P.-L. Taberna, L. Dubois, O. Crosnier, P. Simon, D. Lee, G. De Paëpe and F. Duclairoir, ACS Nano, 2019, 13, 1443-1453.

65 H. Banda, B. Daffos, S. Périé, Y. Chenavier, L. Dubois, D. Aradilla, S. Pouget, P. Simon, O. Crosnier, P.-L. Taberna and F. Duclairoir, Chem. Mater., 2018, 30, 3040-3047.

66 B.-A. Mei, O. Munteshari, J. Lau, B. Dunn and L. Pilon, J. Phys. Chem. C, 2018, 122, 194-206.

67 H. Koga, H. Tonomura, M. Nogi, K. Suganuma and Y. Nishina, Green Chem., 2016, 18, 1117-1124.

68 C. Wan, Y. Jiao and J. Li, J. Mater. Chem. A, 2017, 5, 38193831.

69 F. Barzegar, A. Bello, O. Guellati, D. Momodu, A. Harat, J. Dangbegnon, M. Guerioune and N. Manyala, Electrochim. Acta, 2015, 186, 277-284.

70 K. Gao, Z. Shao, J. Li, X. Wang, X. Peng, W. Wang and F. Wang, J. Mater. Chem. A, 2013, 1, 63-67.

71 Y. Zhang, Z. Shang, M. Shen, S. P. Chowdhury, A. Ignaszak, S. Sun and Y. Ni, ACS Sustain. Chem. Eng., 2019, 7, 1117511185.

72 K. Ghosh and C. Y. Yue, Electrochim. Acta, 2018, 276, 47-63. 73 Y. Yoon, K. Lee, S. Kwon, S. Seo, H. Yoo, S. Kim, Y. Shin, Y. Park, D. Kim, J.-Y. Choi and H. Lee, ACS Nano, 2014, 8, 4580-4590.

74 R. K. Pal, S. C. Kundu and V. K. Yadavalli, ACS Appl. Mater. Interfaces, 2018, 10, 9620-9628. 